\title{
Fully constrained linear spectral unmixing based global shadow compensation for high resolution satellite imagery of urban areas
}

Yang, Jian \& He, Yuhong \& Caspersen, John

Version Post-print/Accepted Manuscript

Citation Yang, J., He, Y., \& Caspersen, J. (2015). Fully constrained linear (published version) spectral unmixing based global shadow compensation for high resolution satellite imagery of urban areas. International Journal of Applied Earth Observation and Geoinformation, 38, 88-98. $\underline{10.1016 / \mathrm{j} \cdot j \mathrm{jag} \cdot 2014.12 .005}$.

Copyright/License

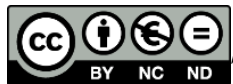
Attribution-NonCommercial-NoDerivatives 4.0 International License. To view a copy of this license, visit Creative Commons BY NC ND 4.0 License.

How to cite TSpace items

Always cite the published version, so the author(s) will receive recognition through services that track citation counts, e.g. Scopus. If you need to cite the page number of the author manuscript from TSpace because you cannot access the published version, then cite the TSpace version in addition to the published version using the permanent URI (handle) found on the record page.

This article was made openly accessible by $U$ of $T$ Faculty. Please tell us how this access benefits you. Your story matters. 
1 Fully constrained linear spectral unmixing based global shadow compensation for

$7{ }^{1}$ Department of Geography, University of Toronto, 100 St. George Street, Toronto, ON M5S

$83 G 3$, Canada.

$9{ }^{2}$ Department of Geography, University of Toronto Mississauga, 3359 Mississauga Rd North, 10 Mississauga, ON L5L 1C6, Canada.

$11{ }^{3}$ Faculty of Forestry, University of Toronto, 33 Willcocks Street, Toronto, ON M5S 3B3, Canada.

12

$13 *$ Corresponding author. Tel.: +1 4169783375.

14 Email address: jiangeo.yang@mail.utoronto.ca (J. Yang).

15

16

17

18

19

20

21

22

23 


\section{Abstract}

Shadows commonly exist in high resolution satellite imagery, particularly in urban areas,

27 which is a combined effect of low sun elevation, off-nadir viewing angle, and high-rise buildings.

28 The presence of shadows can negatively affect image processing, including land cover

29 classification, mapping, and object recognition due to the reduction or even total loss of spectral information in shadows. The compensation of spectral information in shadows is thus one of the most important preprocessing steps for the interpretation and exploitation of high resolution satellite imagery in urban areas. In this study, we propose a new approach for global shadow compensation through the utilization of fully constrained linear spectral unmixing. The basic assumption of the proposed method is that the construction of the spectral scatter plot in shadows is analogues to that in non-shadow areas within a two-dimension spectral mixing space. In order to ensure the continuity of land covers, a smooth operator is further used to refine the restored

37 shadow pixels on the edge of non-shadow and shadow areas. The proposed method is validated using the WorldView-2 multispectral imagery collected from downtown Toronto, Ontario,

39 Canada. In comparison with the existing linear-correlation correction method, the proposed method produced the compensated shadows with higher quality. unmixing, spectral mixing space, spectral scatter plot, WorldView-2. 
1 Introduction

High spatial resolution satellite imagery, such as IKONOS, QuickBird, GeoEye, and

50 WorldView, exhibits enough detailed features to distinguish small objects of land cover in urban

51 areas. Unfortunately, the combined effect of low sun elevation, off-nadir viewing angle, and

52 high-rise buildings results in the ubiquity of shadows in high resolution satellite imagery of

53 urban areas. The reduction or even total loss of spectral information under shadow areas

54 obstructs the image interpretation. It is thus of great importance to restore shadow information

55 before any image processing step (e.g., classification, mapping, and object recognition) is

56 applied.

57 In the workflow of shadow compensation, shadow detection is usually conducted first

58 (Shahtahmassebi et al., 2013), because the quality of shadow detection can directly influence the

59 outcome of shadow restoration ( $\mathrm{Li}$ et al., 2014). Two strategies have been reported in the

60 literature to detect shadows; pixel-based and region-based strategies (Zhou et al., 2009). The

61 former regards individual pixels as the basic unit to determine whether it is a shadow pixel,

62 whereas the latter views a piece of shadow as a continuous region. Given the small size of pixel

63 cells in high spatial resolution imagery, shadow pixels tend to aggregate together as a patch or a

64 cluster. In this case, the pixel-based shadow detection may induce "salt and pepper" noise in a

65 continuous piece of shadow, further resulting in the discontinuity of land covers in restored

66 shadow areas. On the other hand, the region-based strategy has been more preferred and

67 frequently used by recent studies (Dare, 2005; Lorenzi et al., 2012; Massalabi et al., 2004). Most

68 of the region-based shadow detection methods are implemented through the refinement of pixel-

69 based detection using a local morphological filtering operator (Dare, 2005; Lorenzi et al., 2012), 
while very few studies detect shadow regions by integrating imagery segmentation and

71 classification within the framework of object-based image analysis (Massalabi et al., 2004).

72 Unlike pixel-based refining methods, object-based shadow detection is able to make full use of

73 spectral, textural, and contextual features to separate non-shadow and shadow areas (Massalabi

74 et al., 2004).

75 Shadow restoration is the core component of shadow compensation. There are two types

76 of shadow restoration strategies in terms of the processing extent, namely local and global

77 strategies. The local strategy restores spectral information of a shadow pixel or region based on

78 the neighbouring non-shadow land covers (Massalabi et al., 2004), whereas the global strategy

79 handles all the shadows in the imagery in their entirety (Chen et al., 2007; Dare, 2005; Lorenzi et

80 al., 2012; Sarabandi et al., 2004). In most cases, the local strategy can produce a better shadow-

81 restored result because every shadow pixel or region is restored separately. Nevertheless, it is

82 very time consuming to apply the local strategy for a large urban scene. In contrast, the global

83 strategy is more easily implemented, although not all the shadow pixels or regions are well

84 restored. A common basic assumption for both the local and global strategy is that the spectra of

85 land covers in shadow areas are highly related to those in non-shadow areas and the relations

86 between them can be detected through a computation method (e.g., gamma correction (Massalabi

87 et al., 2004; Sarabandi et al., 2004), histogram matching (Dare, 2005; Li et al., 2005; Lorenzi et

88 al., 2012; Sarabandi et al., 2004; Tsai, 2006), and linear-correlation correction (Chen et al., 2007;

89 Sarabandi et al., 2004; Yamazaki et al., 2009; Zhou et al., 2009)). Following this approach, this

90 study assumes that the construction of the spectral scatter plot in shadow areas is analogous to

91 that in non-shadow areas within a two-dimension spectral mixing space. 
In this paper, we propose a fully constrained linear spectral unmixing based global

93 shadow compensation approach for high spatial resolution satellite imagery of urban areas based

94 on the above assumption. The remainder of this paper is organized as follows. In the second

95 section, the study area and dataset is described. Details of the proposed shadow compensation approach are given in the third section. The fourth and fifth sections demonstrate the

97 experimental results and discussion, respectively. The main conclusions are drawn in the last section.

\section{Study area and dataset}

Located in Toronto, Ontario, Canada, the study area is a typical urban land, characterized 102 by a complex mosaic of different land cover types, including street trees, grass, and many highrise buildings. WorldView-2 original multispectral imagery of the central Toronto urban area

104 was adopted for the experiment. It contains eight spectral bands with $2 \mathrm{~m}$ resolution (coastal, 105 blue, green, yellow, red, red edge, near infrared 1 (NIR 1), and near infrared 2 (NIR 2)). Detailed wavelength information of each spectral band is listed in Table 1 . In this study, a $2 \times 2 \mathrm{~km}$ subset 107 of the original dataset of $1000 \times 1000$ pixels was selected (Fig. 1).

The satellite imagery was acquired at 16:42 on June 2nd, 2011 and the solar zenith angle was approximately 22.4 degrees while the satellite zenith angle was approximately 9.0 degrees.

110 It is also worth noting that this experimental area is the downtown Toronto where many

111 buildings are over $20 \mathrm{~m}$ height. Thus, the combination of the low sun elevation, off-nadir 112 viewing angle, and high-rise buildings resulted in the abundance of large shadow areas, which 113 makes it suitable to test the proposed shadow compensation approach. 
Table 1. Wavelength range of each spectral band in the WorldView-2 multispectral imagery.

\begin{tabular}{c|cccccccc}
\hline $\begin{array}{c}\text { Spectral } \\
\text { band }\end{array}$ & Coastal & Blue & Green & Yellow & Red & $\begin{array}{c}\text { Red } \\
\text { edge }\end{array}$ & NIR 1 & NIR 2 \\
\hline $\begin{array}{c}\text { Wavelen } \\
\text { gth (nm) }\end{array}$ & $400-450$ & $450-510$ & $510-580$ & $585-625$ & $630-690$ & $705-745$ & $770-895$ & $860-900$ \\
\hline
\end{tabular}

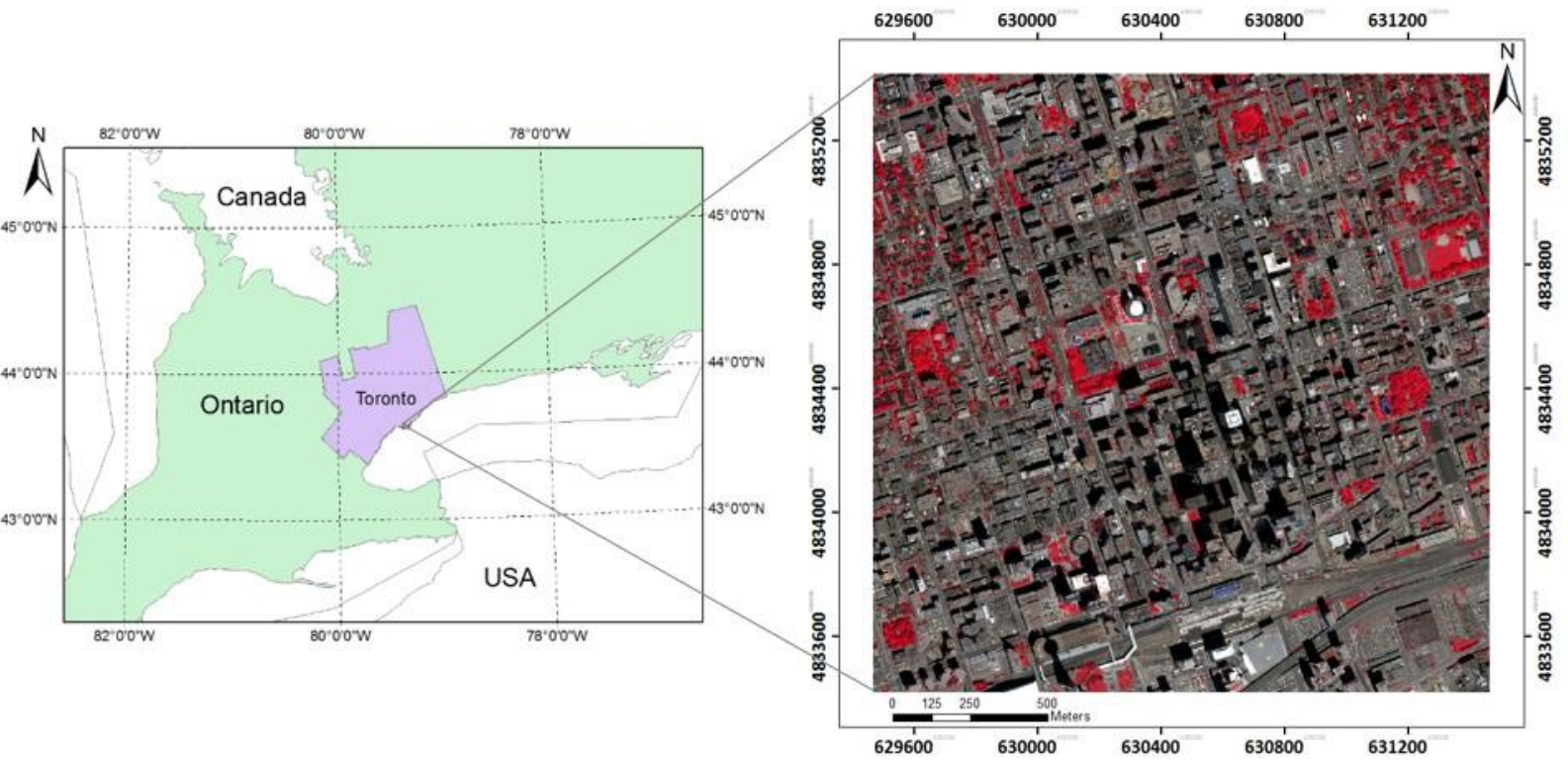

Fig. 1. Location of the study area in downtown Toronto, Ontario, Canada and WorldView-2 multispectral imagery used for experiment and analysis (Band NIR 1, red, and green as R, G, and B). This subset is under WGS84 UTM coordinate system.

\section{Methods}

The processing steps of the proposed global shadow compensation method are provided

124 in the flowchart (Fig. 2). The non-shadow and shadow areas were first separated from the

125 multispectral imagery through the utilization of supervised object-based shadow detection. The

126 endmembers were thereafter identified and optimized using the automated endmember searching

127 method in the non-shadow and shadow areas, respectively. Employing the fully constrained

128 linear spectral unmixing, the fractions of land covers in the shadow area were unmixed by the 
129 optimized endmembers from the shadow area. Using unmixed fractions, the spectra of land

130 covers in the shadow area were further restored on the basis of endmembers optimized from the

131 non-shadow area. Then, the edge of non-shadow and shadow areas was smoothed to refine the

132 shadow-restored imagery. Finally, a commonly-used linear shadow correction method was also

133 adopted and compared with the proposed global shadow compensation approach to examine the

134 performance.

135

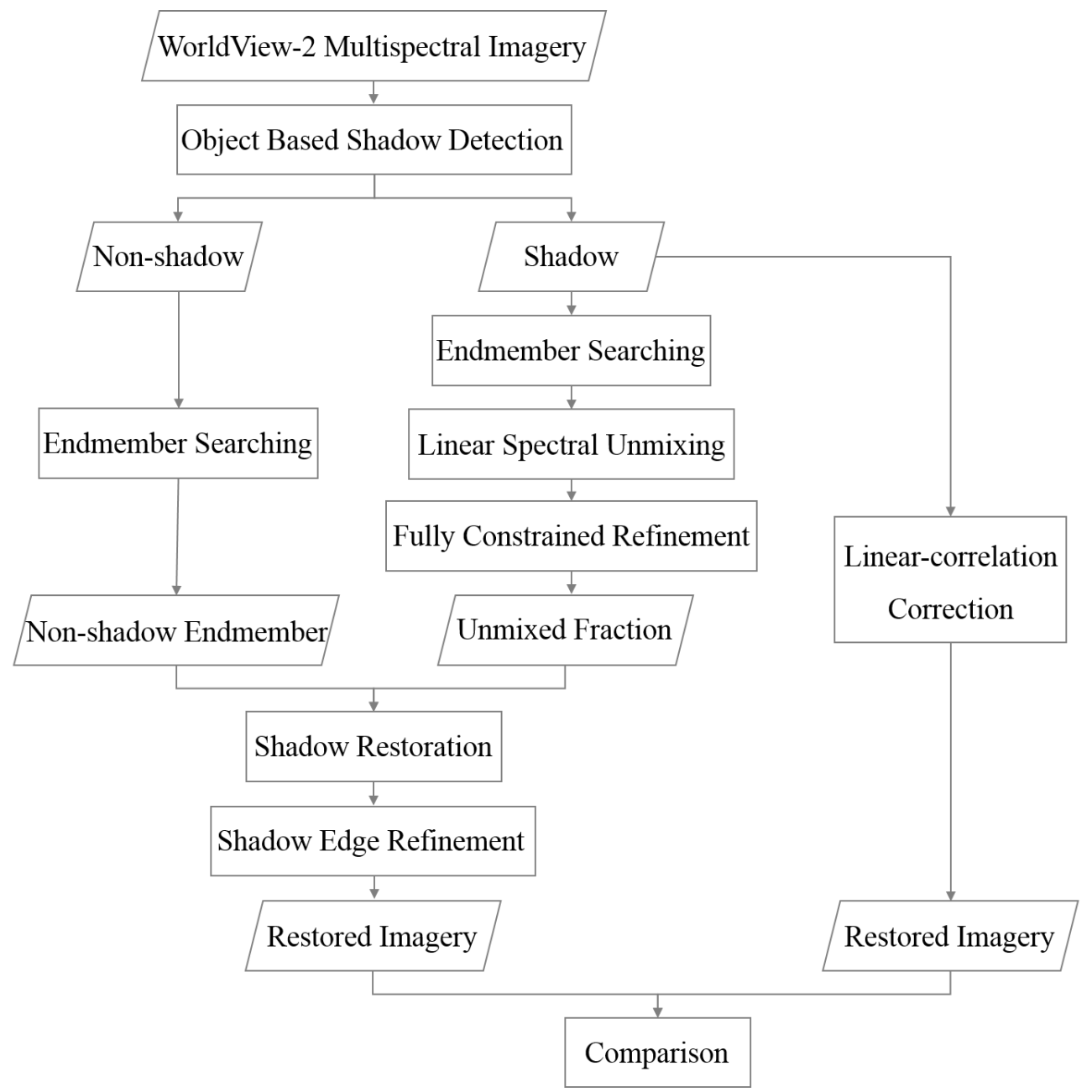

Fig. 2. The processing flowchart of the proposed global shadow compensation method.

3.1 Object-based shadow detection 
It is widely accepted that shadow detection should precede shadow restoration because

141 the quality of shadow detection can directly affect the accuracy of the ultimate shadow-restored

142 imagery. In contrast to the unsupervised histogram threshold method that has been popularized in 143 grayscale or single band of multispectral imagery (Chen et al., 2007; Dare, 2005; Zhou et al.,

144 2009), this study adopted object-based shadow detection using a state-of-the-art supervised

145 classifier, the support vector machine (SVM) (Arbel and Hel-Or, 2011; Lorenzi et al., 2012).

146 Particularly, the BerkeleyImageSeg software package (BIS; http://www.imageseg.com) was

147 implemented to partition the WorldView-2 multispectral imagery into segments of appropriate 148 size for object-based shadow detection. The program utilized the region merging algorithm

149 proposed by (Benz et al., 2004) based on three parameters of threshold, shape (0.1-0.9), and 150 compactness (0.1-0.9). A higher threshold often leads to segments of larger size, whereas higher 151 shape and compactness indicate more round and smooth segments, respectively. After a series of 152 trial-and-error parameter selection, this study chose to use 40, 0.5, and 0.5 as threshold, shape, 153 and compactness for image segmentation. A set of training samples including 50 non-shadow 154 polygons and 50 shadow polygons is manually selected for classification using the radial basis 155 function (RBF) SVM.

$157 \quad 3.2$ Fully constrained linear spectral unmixing based shadow restoration $158 \quad 3.2 .1$ Construction of the spectral scatter plots and automated endmember searching 159 As defined by Small (2003), spectral mixing space is a self-consistent basis for providing 160 the description of urban surface material characteristics. All the pixels in the entire imagery are 161 dotted, and form a spectral scatter plot within a specific spectral mixing space (Small, 2003). 162 Moreover, the construction of spectral scatter plots can vary a lot in different spectral mixing 
163 spaces. Due to the similarity and continuity of land covers between the non-shadow and shadow

164 areas, the construction of the spectral scatter plot in the shadow area should be very analogous to

165 that in the non-shadow area. We hypothesized that the spectral scatter plots of the non-shadow

166 and shadow areas should demonstrate the same construction or shape but differ in size. Thus, the

167 proposed shadow compensation was implemented by restoring the size of the spectral scatter plot

168 in the shadow area to the size of the spectral scatter plot in the non-shadow area, while also

169 retaining its shape.

170 Compared with the vegetation-impervious-soil model proposed by Ridd (1995), the

171 vegetation-high albedo-low albedo model is more suitable for linear spectral mixing of high

172 resolution urban imagery (Small, 2003; Yang et al., 2014). While impervious surfaces in urban

173 areas become separated as bright and dark surface materials with decreasing spatial resolution,

174 soil can also be categorized into high albedo surface (Yang et al., 2014). Therefore, vegetation,

175 high albedo, and low albedo become widely used as endmembers for linear spectral unmixing of

176 high resolution urban imagery (Small, 2003; Yang et al., 2014).

177 The quality of linear spectral unmixing is largely determined by the endmember selection.

178 Due to the endmember variability, multiple endmember spectral mixture analysis (MESMA) has

179 attracted growing attention in spectral analysis (Somers et al., 2011; Zare and Ho, 2014).

180 However, Rogge et al. (2006), Veraverbeke et al. (2012), and Yang et al. (2014) indicated that

181 applying MESMA takes longer and may not always provide better results. Specifically, Yang et

182 al. (2014) proved that a triangle should be the optimal construction of the spectral scatter plot for

183 linear spectral unmixing in a two-dimension spectral mixing space, and the vertices of this

184 triangle should be the optimal three endmembers. From this perspective, it is of great necessity

185 and significance to construct an appropriate two-dimension spectral mixing space where the 
186 shape of the spectral scatter plot is a triangle and three vertices represent the vegetation, high 187 albedo, and low albedo endmembers, respectively.

Apparently, the endmember of low albedo is located close to the coordinate origin in any

189 spectral mixing space due to the lowest digital number (DN) values in all the spectral bands. In

190 addition, the NIR 1 and NIR 2 bands in the WorldView-2 dataset are considered to be the

191 appropriate spectral bands for vegetation representation and the other bands are able to

192 characterize high albedo land covers. In order to select the appropriate two-dimension spectral

193 mixing space, we combined one spectral band from the coastal, blue, green, yellow, red, and red

194 edge bands, with the other one from the NIR 1 and NIR 2 bands for constructing 12 alternatives.

195 These spectral mixing spaces in the non-shadow and shadow areas are separately presented in

196 Fig. 3.

197
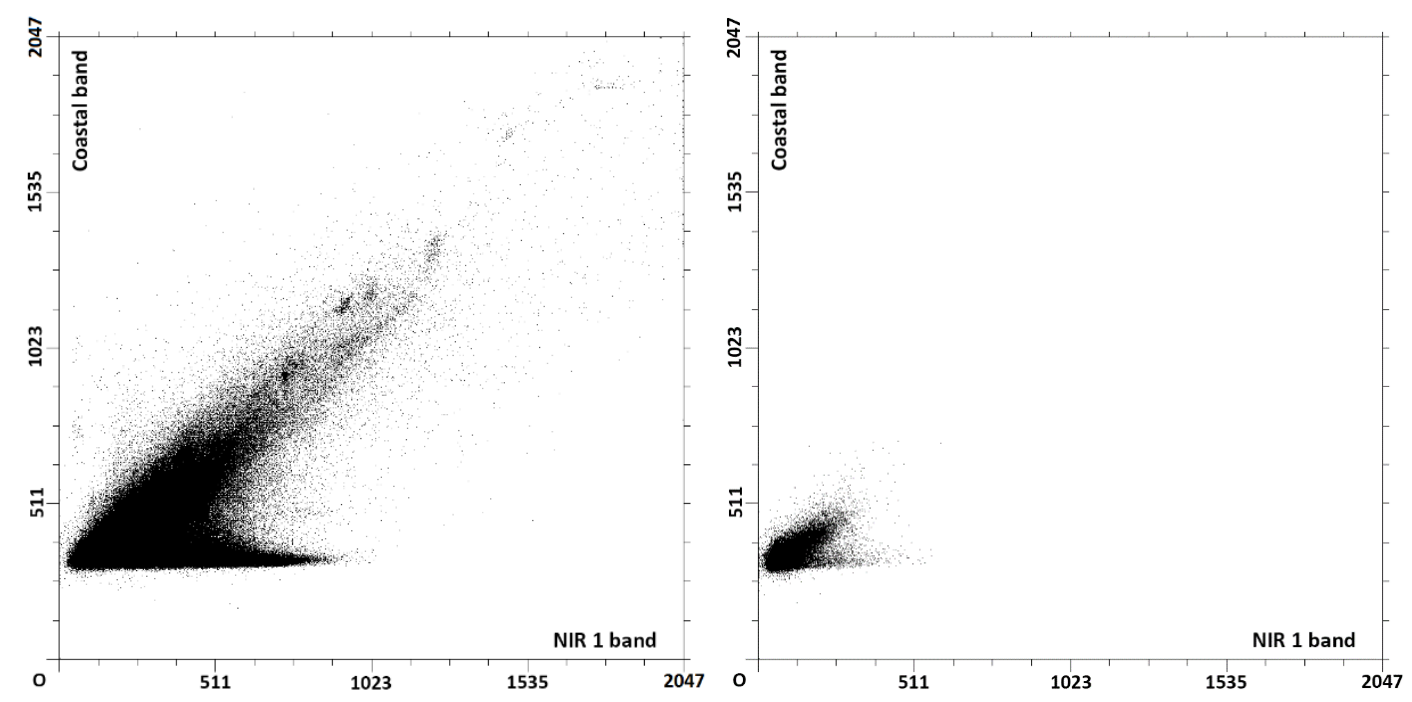

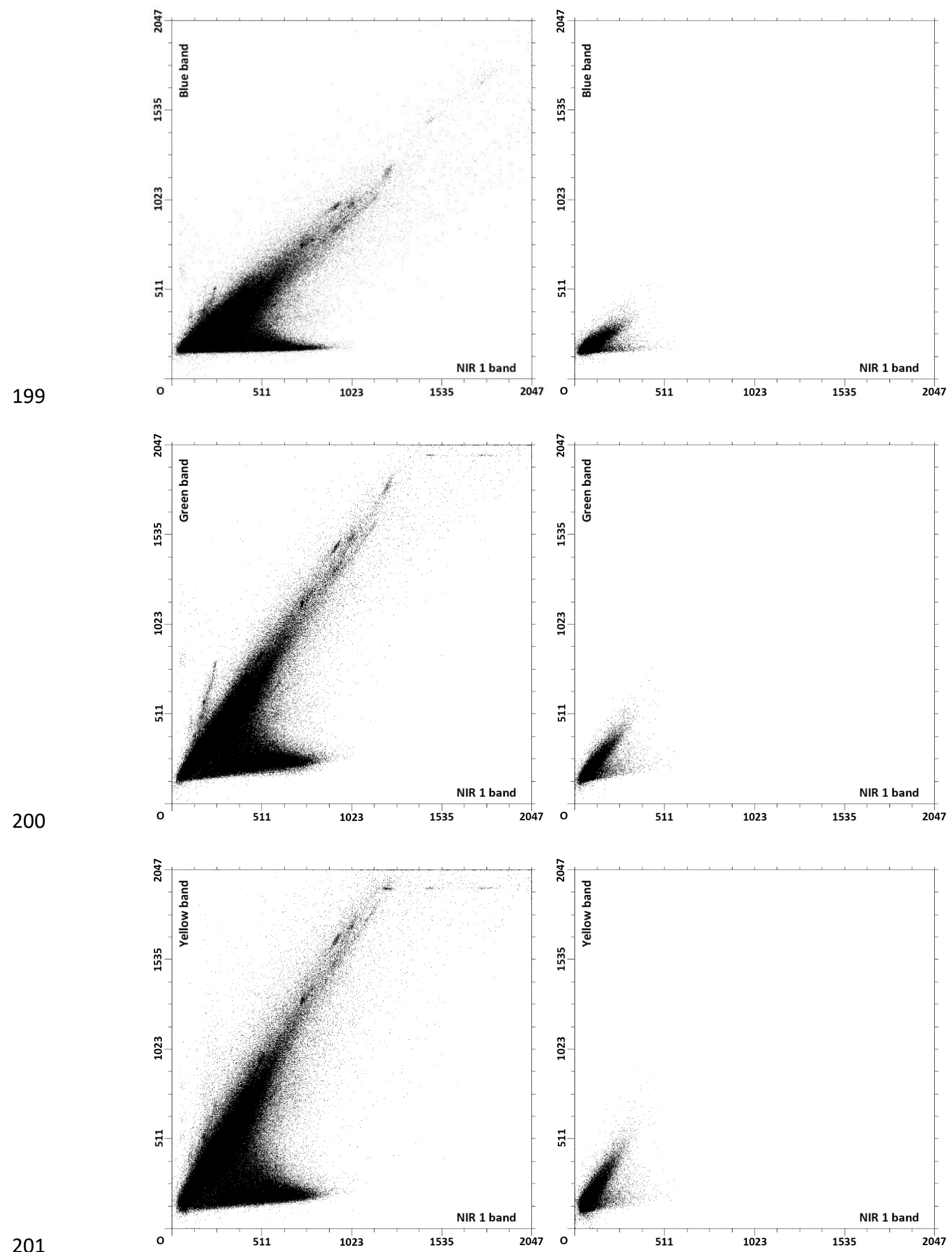

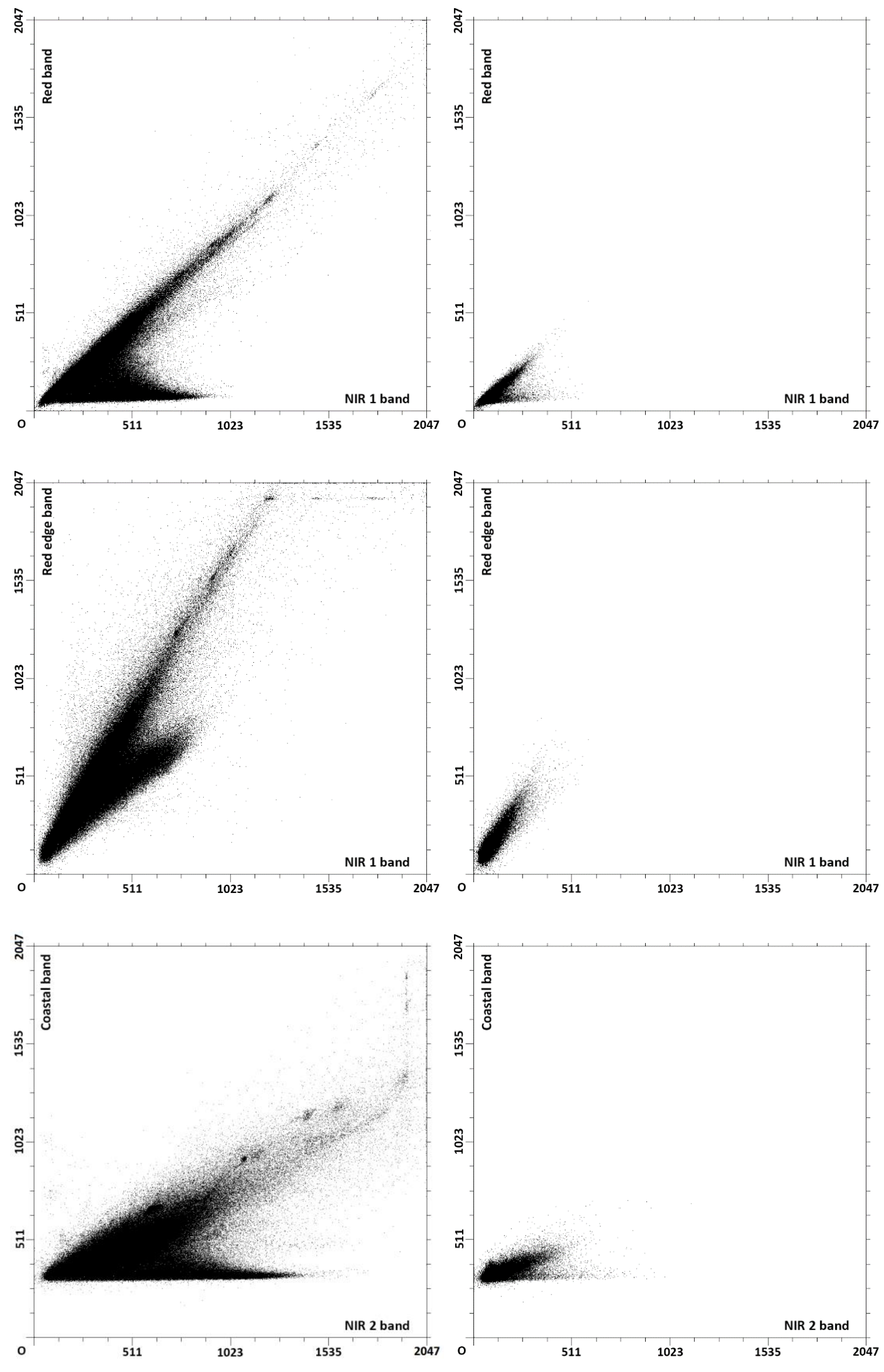

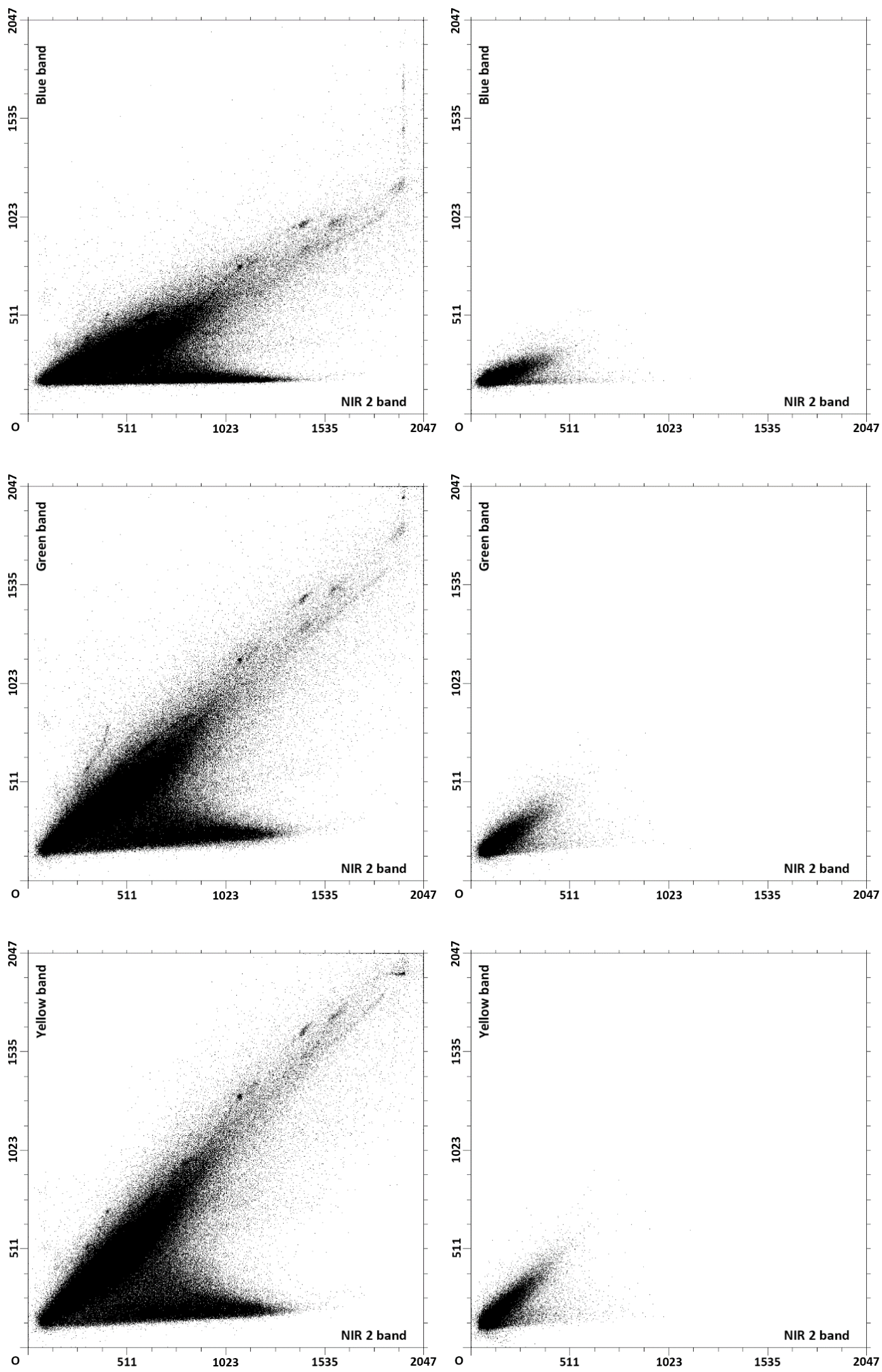

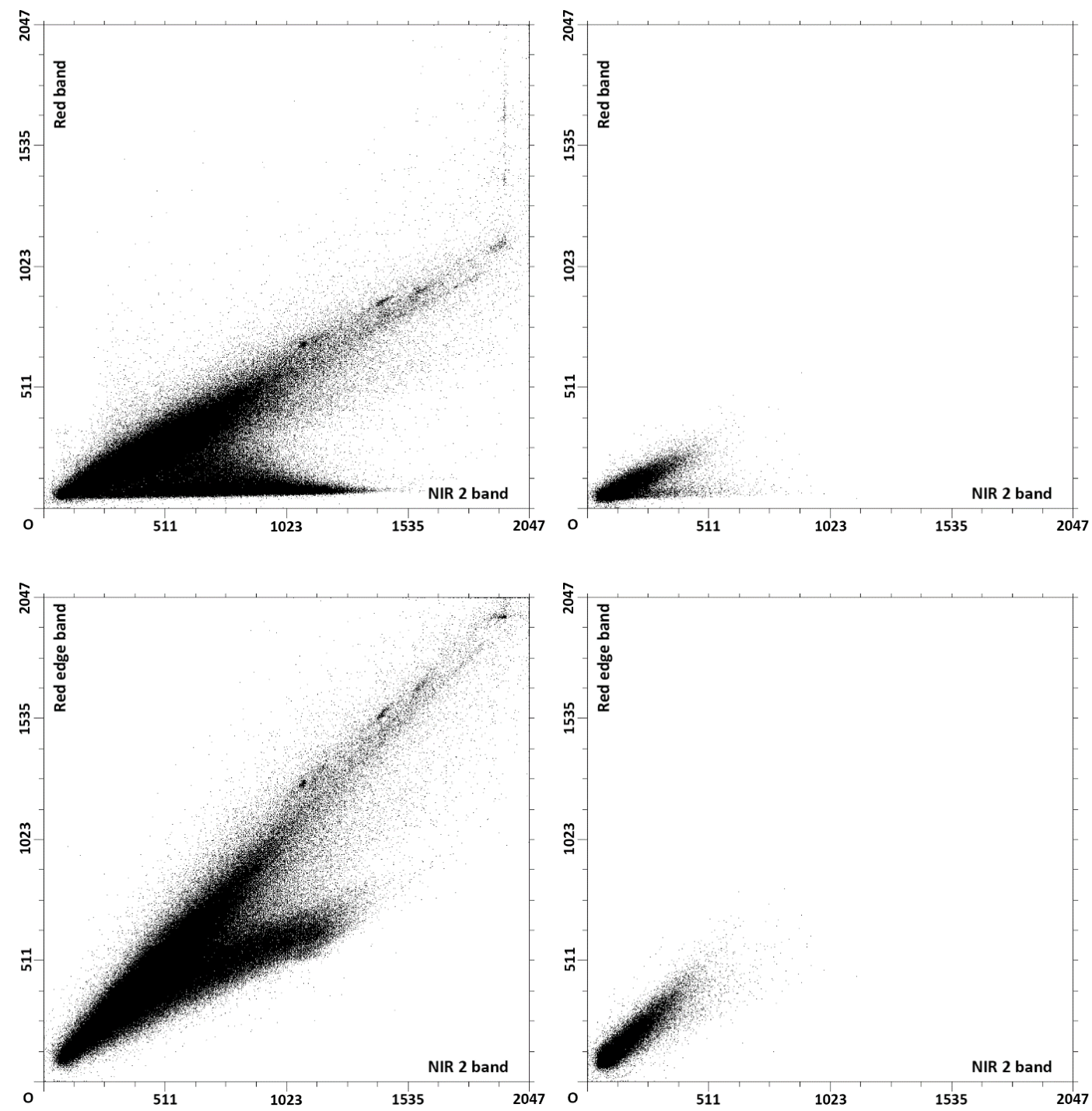

210 Fig. 3. Different spectral mixing spaces constructed with various bands (Coastal, Blue, Green,

211 Yellow, Red, Red edge, NIR 1, NIR 2) in the non-shadow (left) and shadow (right) areas.

It was shown by Small (2003) and Yang et al. (2014) that the straight edges between the

214 vertices of a triangle can result in a valid and meaningful linear spectral unmixing. In contrast to

215 the NIR 2 band, the spectral scatter plots on the basis of the NIR 1 band construct better triangles

216 with straight and distinct edges in both the non-shadow or shadow areas (Fig. 3). Moreover, the

217 spectral mixing space constructed by the red and NIR 1 bands is believed to have the capability 
218 to produce the best quality of linear spectral unmixing among the six spaces on the basis of NIR

2191 band (Fig. 3). Within this spectral mixing space, the highest similarity of the triangles between

220 the non-shadow and shadow areas further ensures that it is the best spectral mixing space to

221 identify the optimal endmembers in both the non-shadow \& shadow areas, and to unmix the

222 spectra of pixels in the shadow area. However, the linear spectral unmixing in this two-

223 dimension spectral mixing space is not able to provide endmember fractions for more than two

224 spectral bands. To obtain a multispectral shadow-restored imagery, the same procedure is also

225 implemented within another two-dimension spectral mixing space constructed by the green and

226 NIR 1 bands because the green band is another component of the standard false color composite

227 in addition to the red and NIR bands. Meanwhile, the spectral scatter plots also indicate good

228 triangles for the non-shadow and shadow areas within the green-NIR 1 spectral mixing space 229 (Fig. 3).

230 In the spectral mixing space constructed by the red and NIR 1 bands (non-shadow \& 231 shadow shown in Fig. 3), the vertices of the scatter plot triangle represent the vegetation, high

232 albedo, and low albedo endmembers. It is worth noting that very dark pixels exist in the shadow 233 area but also scatter in the non-shadow area because the shadow area was detected based on 234 spectral properties of segmented land cover objects rather than those of individual pixels. Hence, 235 the endmember of low albedo is the vertex which locates the closest to the coordinate origin $(\mathrm{O})$ 236 for no matter the non-shadow or shadow area. Besides that, the vertex of the triangle close to the 237 axis of NIR 1 band represents the vegetation endmember while the other vertex close to the axis 238 of red band represents the high albedo endmember. In order to search for these three vertices, an 239 endmember spatial measure index $\left(E S M_{n}\right)$ was newly proposed by Yang et al. (2014) from the 240 perspective of avoiding endmember collinearity and multi-collinearity (Van Der Meer and De 
241 Jong, 2000; Van der Meer and Jia, 2012). As concluded by Yang et al. (2014), the higher value

242 of $E S M_{n}$ indicates a better endmember combination for linear spectral unmixing. Specifically,

$243 E S M_{n}$ can be denoted as $E S M_{2}$ in the two-dimension spectral mixing space constructed by the red

244 and NIR 1 bands, which is directly proportional to the determinant (Det) of two endmember

245 vectors,

246

$$
E S M_{2} \propto \operatorname{Det}\left(\overrightarrow{E M_{V}}-\overrightarrow{E M_{L}} \quad \overrightarrow{E M_{H}}-\overrightarrow{E M_{L}}\right)(1)
$$

247 , where $\overrightarrow{E M_{V}}, \overrightarrow{E M_{H}}$, and $\overrightarrow{E M_{L}}$ represent the endmember vectors of vegetation, high albedo, 248 and low albedo, respectively.

We can notice from Eq. (1) that the value of $E S M_{2}$ is proportional to the area of the 250 triangle constructed by these three vertices (endmembers). The best endmember combination is 251 in effect corresponding to the largest area of the triangle among all the triangles constructed by 252 any three vertices. Considering one of the triangle vertices (low albedo endmember) is fixed to 253 the pixel nearest the coordinate origin, we need to search the other two vertices (vegetation and 254 low albedo endmembers) from the remaining pixels in order that three vertices constructs a 255 triangle with the largest area. Nevertheless, it will be too time consuming if calculating the $E_{S} M_{2}$ 256 for every pair of two pixels, particularly in the case of a huge experimental area. Thus, this study 257 developed a rapid algorithm to automatically search for three endmembers which construct the 258 largest area of triangle. For a cluster of pixels scattered in a two-dimension space, it is not 259 difficult to prove that three pixels that construct the largest triangle area must locate at the 260 convex hull of all the pixels. So we firstly extracted the convex hull for the spectral scatter plot 261 by implementing Graham's scan (Graham, 1972), which is a time-efficient method of computing 262 the convex hull of a finite set of points in the plane. Besides that the endmember of low albedo is 263 determined as the pixel closet to the coordinate origin, the vegetation and low albedo 
264 endmembers were then selected from the remaining pixels at the convex hull. Compared to the

265 calculation for every pair of two pixels, the rapid endmember searching can remarkably decrease

266 the time complexity from $\mathrm{O}\left(\mathrm{n}^{2}\right)$ to $\mathrm{O}(\mathrm{n} \log n)$.

267 Due to the various sizes of the scatter plot triangles (Fig. 3), this study optimized two sets

268 of endmember combinations (vegetation, high albedo, and low albedo) for the non-shadow and

269 shadow areas, respectively. As such, two sets of endmember combinations were also

270 automatically identified within the within the green-NIR 1 spectral mixing space.

$271 \quad 3.2 .2$ Fully constrained linear spectral unmixing

272 As an important branch of soft classification, linear spectral unmixing has been

273 frequently adopted in many remote sensing applications, especially for medium/low resolution

274 imagery. In recent years, several studies have started to unmix fine-scale imagery (e.g., IKONOS

275 and WorldView-2) (Nichol and Wong, 2007; Nichol et al., 2010; Small, 2003; Yang et al., 2014).

276 As mentioned previously, the linear spectral unmixing was adopted to obtain the fractions of

277 vegetation, high albedo, and low albedo for any pixel in the shadow area. Mathematically, linear

278 spectral unmixing in the red-NIR 1 spectral mixing space is a process of solving the linear

279 equations below:

280

$$
\left\{\begin{array}{l}
D N_{R E D}(s)=f_{V} D N_{\text {VRED }}(s)+f_{H} D N_{\text {HRED }}(s)+f_{L} D N_{\text {LRED }}(s) \\
D N_{\text {NIRI }}(s)=f_{V} D N_{\text {VNIRI }}(s)+f_{H} D N_{\text {HNIRI }}(s)+f_{L} D N_{\text {LNIRI }}(s)
\end{array}\right.
$$

281 , where $D N_{R E D}(s)$ and $D N_{N I R I}(s)$ are the $\mathrm{DN}$ values of the unmixed shadow pixel in the red and

282 NIR 1 bands; $f_{V}, f_{H}$, and $f_{L}$ are the fractions of vegetation, high albedo, and low albedo,

283 respectively; $D N_{X R E D}(s)$ and $D N_{X N I R I}(s)$ denote the $\mathrm{DN}$ values for each endmember optimized

284 from the shadow area $(X: V, H, L$, i.e., vegetation, high albedo, and low albedo). Additionally, 
285 the sum of vegetation, high albedo, and low albedo fractions should be equal to one for any

286 given unmixed pixel:

$$
f_{V}+f_{H}+f_{L}=1(3)
$$

To simplify the solution procedure, we translated the scatter plot triangle so that the pixel

289 of low albedo endmember exactly located the coordinate origin. Meanwhile, the optimized

290 vegetation and high albedo endmembers will move towards the coordinate origin corresponding

291 to the offset of the low albedo endmember. The linear translation will not affect the fraction of

292 each endmember (i.e., $f_{V}, f_{H}, f_{L}$ ) as the shape of the scatter plot triangle does not change in

293 this process. Given that the post-translated spectrum of low albedo endmember is assigned as $(0$,

294 0), Eq. (2) can thus be rewritten as:

$$
\left\{\begin{array}{l}
D N_{R E D}^{\prime}(s)=f_{V} D N_{V R E D}^{\prime}(s)+f_{H} D N_{H R E D}^{\prime}(s) \\
D N_{N I R I}^{\prime}(s)=f_{V} D N_{V N I R I}^{\prime}(s)+f_{H} D N_{H N I R I}^{\prime}(s)
\end{array}\right.
$$

296 , where $D N_{R E D}^{\prime}(s)$ and $D N_{N I R I}^{\prime}(s)$ represent the post-translated DN values of the unmixed shadow

297 pixel in the red and NIR 1 bands, whereas $D N_{X R E D}^{\prime}(s)$ and $D N_{X N I R I}^{\prime}(s)$ represent the post-

298 translated DN values for each endmember from the shadow area $(X: V, H$, i.e., vegetation and 299 high albedo). It can also be noticed that Eq. (4) must produce a unique solution when the 300 vegetation and high albedo endmembers are optimized by the largest value of $E S M_{2}$ due to no 301 collinearity of $\overrightarrow{E M_{V}(s)}-\overrightarrow{E M_{L}(s)}$ and $\overrightarrow{E M_{H}(s)}-\overrightarrow{E M_{L}(s)}$. With the calculated $f_{V}$ and $f_{H}$, the 302 fraction of low albedo $\left(f_{L}\right)$ can be easily derived using Eq. (3).

304 fraction should not be over the range of zero to one. The schematic triangle of the post-translated 305 scatter plot in the red-NIR 1 spectral mixing space is shown as Fig. 4. Assuming $E M_{V}(s)$ and 
$E M_{H}(s)$ are the selected pair of pixels from the shadow area as vegetation and high albedo

307 endmembers with the highest value of $E S M_{2}$, it is apparent to notice that the pixels within the

308 triangle $E M_{V}(s) E M_{H}(s) E M_{L}(s)$ have the meaningful fraction of vegetation, high albedo, and

309 low albedo (0-1). Nevertheless, it is not always true that all the pixels are dotted into this triangle

310 even using the optimized endmembers. For any pixel outside of the triangle, this study solved Eq.

311 (4) by searching the point on the boundary of this triangle with the minimum Euclidean distance

312 to the outlier. In particular, the outlier pixels were divided into two zones (Fig. 4). For the pixel

313 in Zone 1, the solution is the closest vertex (i.e., $E M_{V}(s)$ or $E M_{H}(s)$ ), indicating that it is a pure

314 vegetation or high albedo pixel. Besides that, the solution for the pixel in Zone 2 is the closest

315 point on any side of the triangle. This is a necessary step to validate the unmixed fraction of each

316 endmember is ranging from zero to one. Similar to endmember searching, the fully constrained

317 linear spectral unmixing was implemented within both the red-NIR 1 and green-NIR 1 spectral

318 mixing spaces.

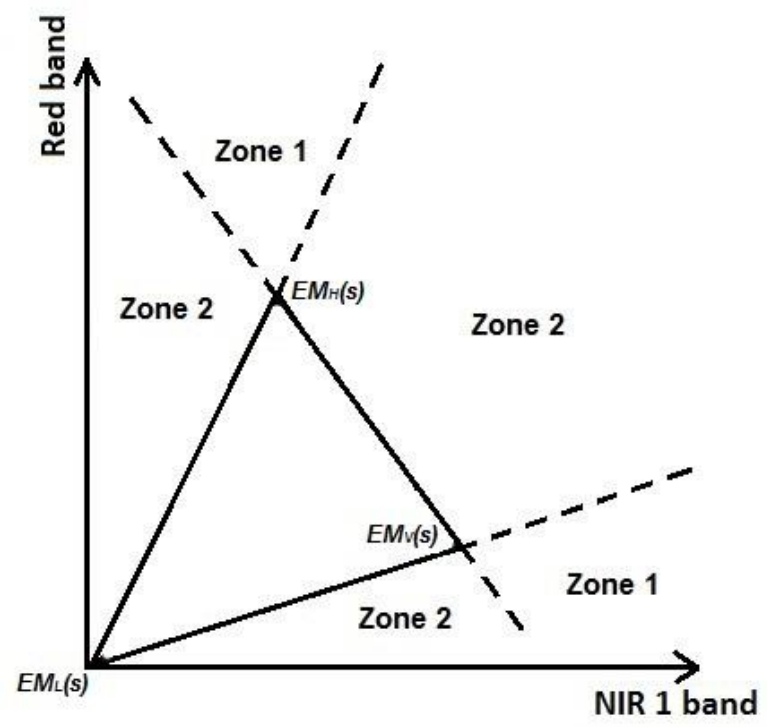


321 Fig. 4. An illustration of schematic scatter plot triangle in the two-dimension spectral mixing

322 space constructed with the red and NIR 1 bands. $E M_{V}(s), E M_{H}(s)$, and $E M_{L}(s)$ represent

323 vegetation, high albedo, and low albedo endmembers optimized from the shadow area.

324

325

326

327

328

329

330

331 332 denoted:

333

$$
\left\{\begin{array}{l}
\Lambda \\
D N_{R E D}=f_{V} D N_{V R E D}(n)+f_{H} D N_{\text {HRED }}(n)+f_{L} D N_{\text {LRED }}(n) \\
\Lambda \\
D N_{\text {NIRI }}=f_{V} D N_{V N I R I}(n)+f_{H} D N_{\text {HNIRI }}(n)+f_{L} D N_{\text {LNIRI }}(n)
\end{array}\right.
$$

334 , where $\stackrel{\Lambda}{D N_{R E D}}$ and $\stackrel{\Lambda}{D N_{N I R I}}$ are the shadow-restored DN values in the red and NIR 1 bands; 336 shadow area $(X: V, H, L$, i.e., vegetation, high albedo, and low albedo).

In addition, the shadow-restored $\mathrm{DN}$ value for the green band ( $\stackrel{\Lambda}{D N_{\text {GREEN }}}$ ) was calculated

\subsubsection{Shadow restoration}

It is observed from the red-NIR 1 spectral mixing space (Fig. 3) that the similarity of scatter plot triangles between the non-shadow and shadow areas provides a great opportunity for spectral restoration in the shadow area by utilizing the spectral information in the non-shadow area. A feasible way is thus to stretch the scatter plot triangle in the shadow area towards the triangle size in the non-shadow area. For any pixel in the shadow area, the fractions of vegetation $\left(f_{V}\right)$, high albedo $\left(f_{H}\right)$ and low albedo $\left(f_{L}\right)$ remain the same and the restored DN values can be

\section{$335 D N_{X R E D}(n)$ and $D N_{X N I R I}(n)$ are the DN values for each endmember optimized from the non-}

through the expansion of the scatter plot triangle in the green-NIR 1 spectral mixing space.

$$
\stackrel{\Lambda}{D N_{G R E E N}}=f_{V}^{\prime} D N_{\text {VGREEN }}(n)+f_{H}^{\prime} D N_{H G R E E N}(n)+f_{L}^{\prime} D N_{L G R E E N}(n)
$$


340 , where $f_{V}^{\prime}, f_{H}^{\prime}$, and $f_{L}^{\prime}$ represent the fractions of vegetation, high albedo, and low albedo in

341 this two-dimension spectral mixing space; $D N_{\text {XGREEN }}(n)$ represents the $\mathrm{DN}$ value for each

342 endmember optimized from the non-shadow area in the green band $(X: V, H, L$, i.e., vegetation,

343 high albedo, and low albedo).

$344 \quad$ 3.2.4 Edge refinement

345 Since object-based shadow detection can always result in the spatial discontinuity of land

346 covers on the edge of non-shadow and shadow areas, we smoothed the shadow edge through the

347 utilization of mean filter in a $3 \times 3$ moving window (Eq. (7)). The shadow edge was detected

348 from the shadow imagery using the Laplacian operator in a $3 \times 3$ moving window (Eq. (8)).

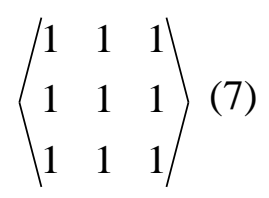

$$
\left\langle\begin{array}{ccc}
0 & -1 & 0 \\
-1 & 4 & -1 \\
0 & -1 & 0
\end{array}\right\rangle(8)
$$

For further comparison, the conventional linear-correlation correction method was

352 utilized for shadow restoration to evaluate the performance of the proposed method. Compared

353 with other shadow restoration techniques (e.g., gamma correction and histogram matching), the

354 linear-correlation correction method was proven more effective (Dare, 2005; Sarabandi et al.,

355 2004). It assumes the signals recoded in the shadow area are still able to provide enough useful

356 information for shadow restoration despite being relatively weak. Specifically, a linear

357 relationship between DN values of the shadow area and those of the non-shadow area for each

358 spectral band (i.e., the green, red, and NIR 1 bands) can be expressed as (Chen et al., 2007;

359 Sarabandi et al., 2004; Yamazaki et al., 2009; Zhou et al., 2009): 


$$
\stackrel{\wedge}{D N}=\frac{\sigma(n)}{\sigma(s)}(D N-\mu(s))+\mu(n)
$$

361 , where $\stackrel{\Lambda}{D N}$ and $D N$ represent the shadow-restored and original DN values of any shadow

362 pixel; $\mu(n)$ and $\mu(s)$ are the mean DN values for the non-shadow and shadow areas while $\sigma(n)$

363 and $\sigma(s)$ are the standard deviation of the non-shadow and shadow areas. In order to eliminate

364 the impact caused by shadow detection, both of them were implemented based on the same

365 object-based detected shadow imagery.

3674 Comparison of methods

In this section, the proposed global shadow compensation approach based on the fully constrained linear spectral unmixing is implemented to produce the shadow-restored imagery, 370 and further visually compared with the mentioned linear-correlation correction method, in terms 371 of the entire imagery and four subsets.

372 The shadow area detected by object-based image classification is shown in Fig. 6. The 373 proportion of shadow area occupies about 15 percent of the entire imagery, which provides 374 enough samples to verify the performance of the proposed compensation method. It is also worth 375 noting that object-based shadow detection successfully extracts patches of shadow area rather 376 than scattered shadow pixels. Since most of the shadows exist as the form of patches in high 377 resolution imagery, the object-based detected shadow area ensures the homogeneity of land 378 covers within each shadow patch.

379 The vegetation, high albedo, and low albedo endmembers are automatically optimized by 380 the proposed rapid endmember searching method in two spectral mixing spaces (i.e., Red + NIR 3811 bands and Green + NIR 1 bands). Moreover, two sets of endmembers (Table 2) are identified 
382 in the non-shadow and shadow areas, respectively. Since all the endmembers of low albedo

383 locate at the coordinate origin $(\mathrm{O})$, we don't have to translate the scatter plot triangle in this

384 experiment. Due to the common spectral basis (i.e., NIR 1 band), the vegetation endmembers

385 selected from these two spectral mixing spaces are identical for both the non-shadow $(1033,75$,

386 and 278 as NIR 1, Red, and Green bands) and shadow areas (547, 61, and 192 as NIR 1, Red,

387 and Green bands). However, the high albedo endmembers optimized within these two spaces are

388 very different from each other. In the shadow area, the fraction of vegetation, high albedo, and

389 low albedo endmembers are derived by the described fully constrained linear spectral unmixing

390 method. For the Red + NIR 1 and Green + NIR 1 spectral mixing spaces, a subset of vegetation,

391 high albedo, and low albedo fraction maps are depicted, respectively (Fig. 5). Thereafter, the

392 shaded spectra of the NIR 1, red, and green bands are recovered using Eq. (5) and (6), as shown

393 in Fig. 6 with a band combination of standard false color composite.

Table 2. Vegetation, high albedo, and low albedo endmembers optimized from two spectral mixing spaces (Red + NIR 1 and Green + NIR 1) for the non-shadow and shadow areas.

\begin{tabular}{c|cccc}
\hline \multirow{2}{*}{ Endmember } & \multicolumn{2}{|c}{ Non-shadow area } & \multicolumn{2}{c}{ Shadow area } \\
& Red + NIR 1 & Green + NIR 1 & Red + NIR 1 & Green + NIR 1 \\
\hline Vegetation & $(75,1033)$ & $(278,1033)$ & $(61,547)$ & $(192,547)$ \\
High albedo & $(2047,2047)$ & $(2047,1298)$ & $(478,471)$ & $(771,443)$ \\
Low albedo & $(0,0)$ & $(0,0)$ & $(0,0)$ & $(0,0)$ \\
\hline
\end{tabular}




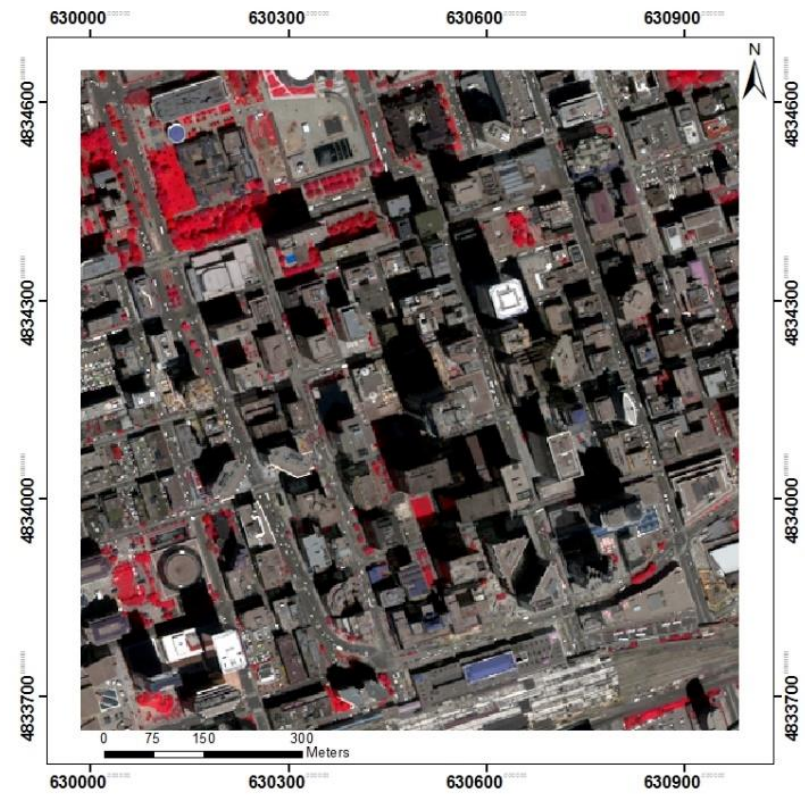

(A)

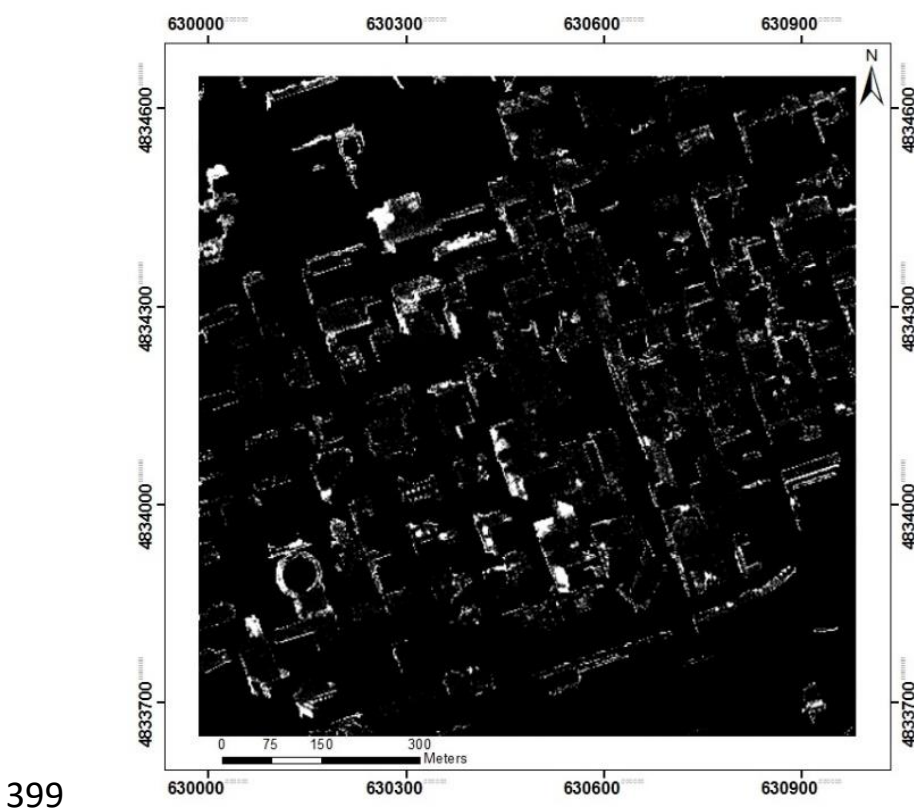

(B)

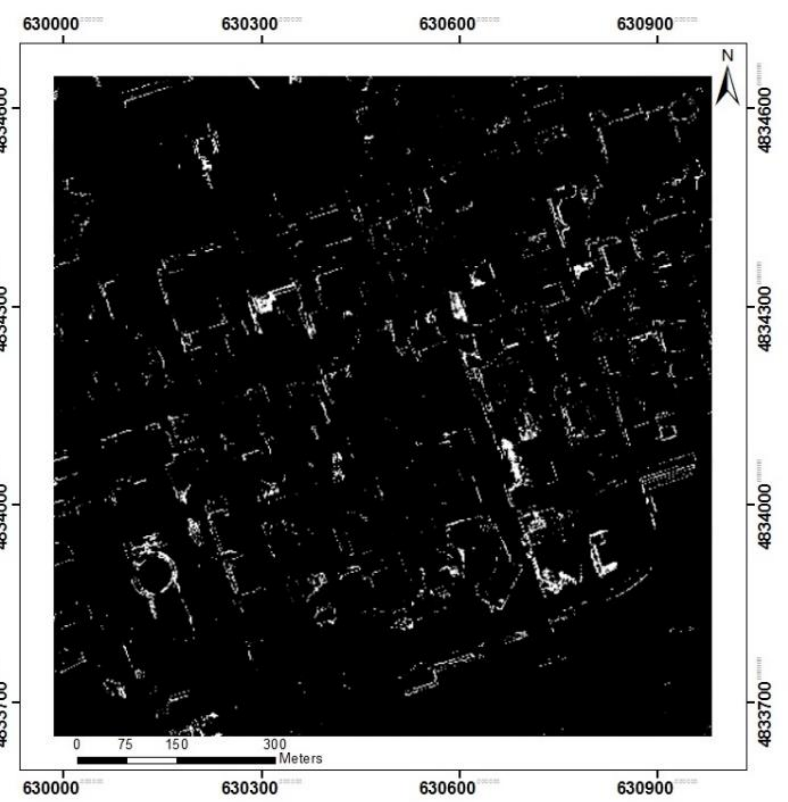

(C) 


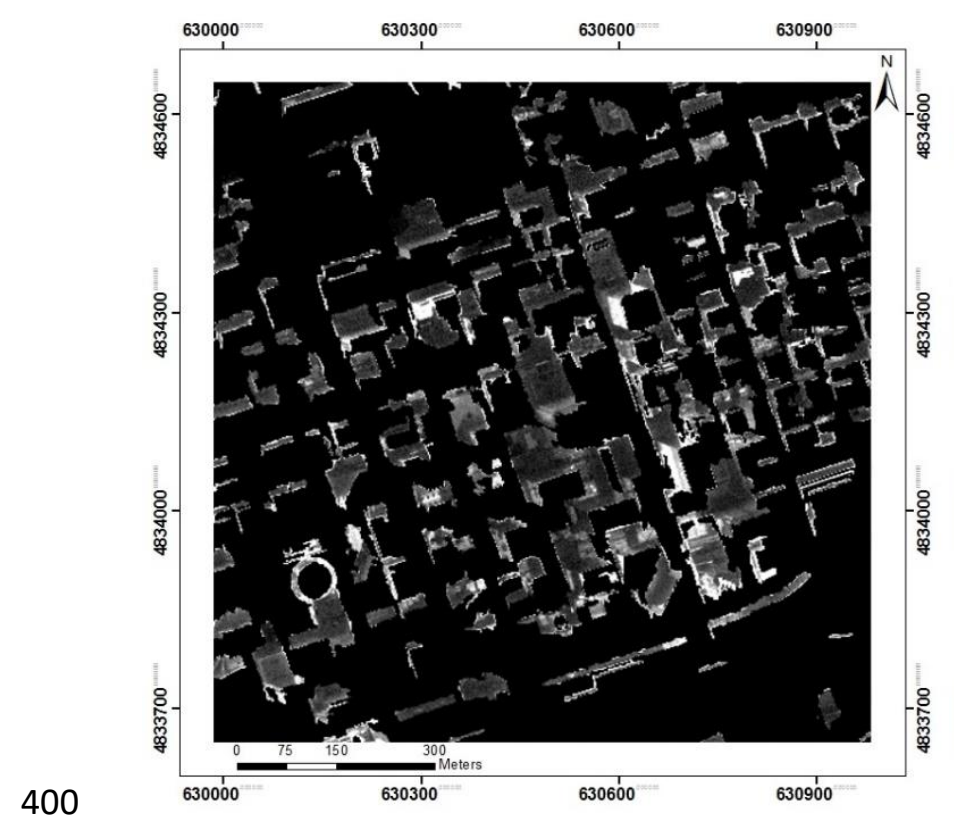

(D)

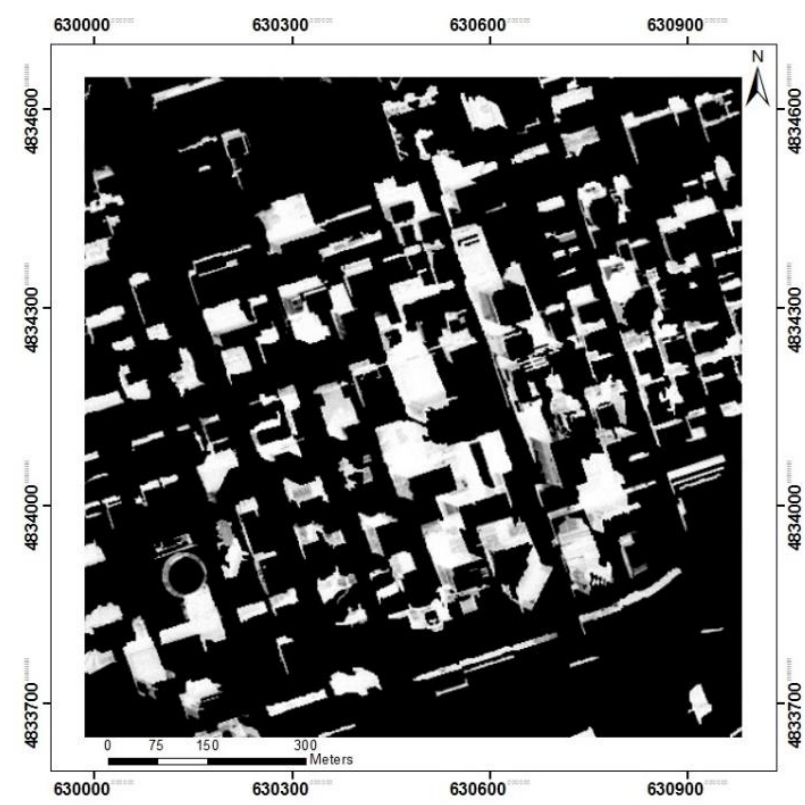

(F)

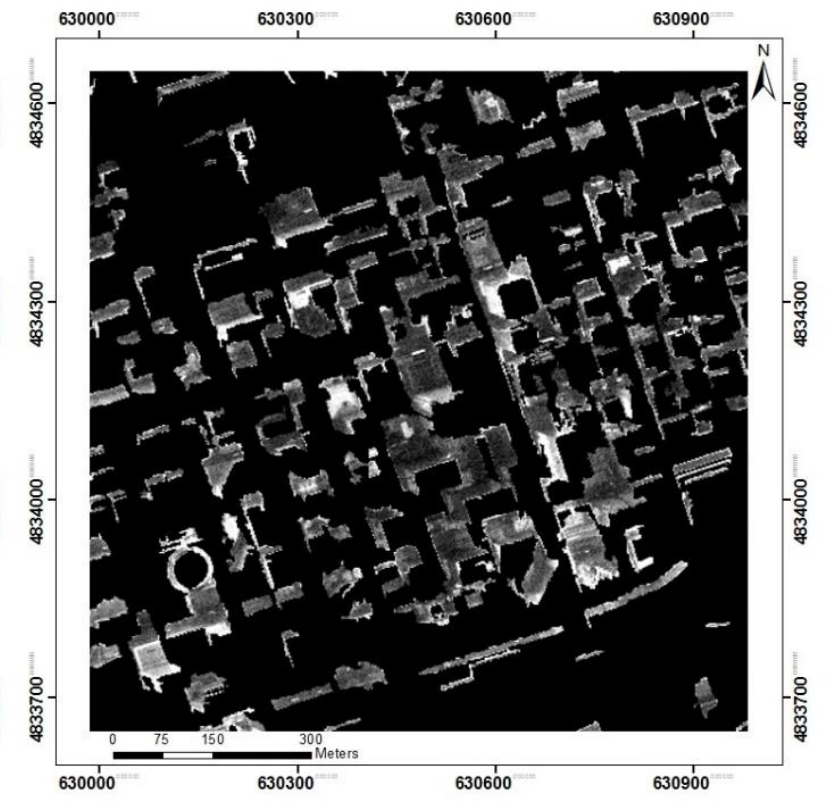

(E)

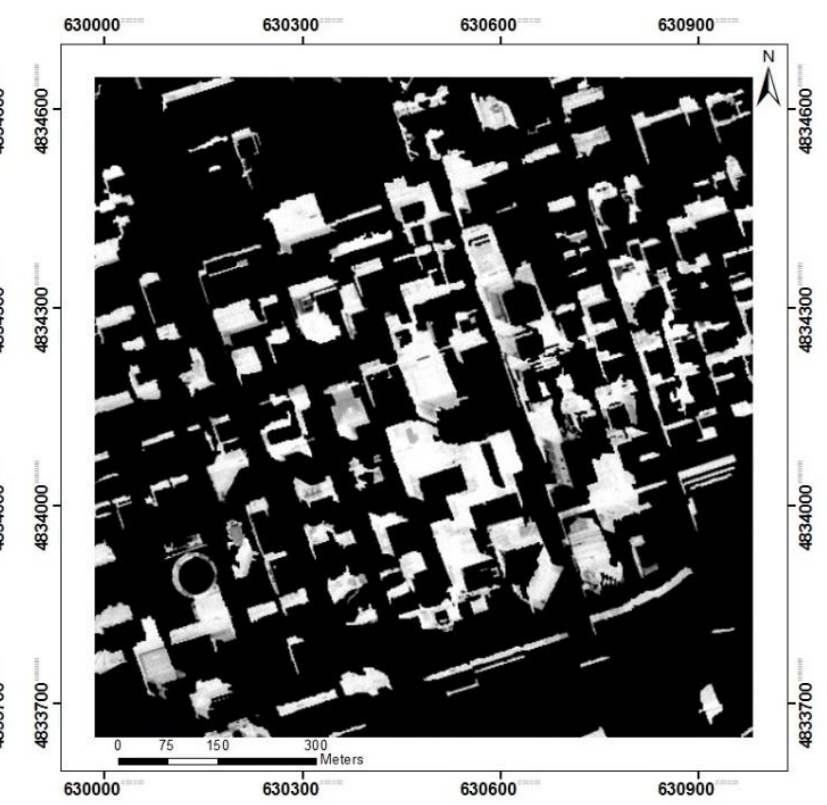

$(\mathrm{G})$

402 Fig. 5. An illustration of original multispectral imagery (A) and fraction maps (B-G) for a subset

403 area where shadows are dominant, including vegetation (B and C), high albedo (D and E), and

404 low albedo (F and G). The fraction maps (0-1) are derived within the Red + NIR 1 (B, D, and F)

405 and Green + NIR $1(\mathrm{C}, \mathrm{E}$, and G) spectral mixing spaces, respectively. 
408 restoration as a comparative experiment. Similarly, the spectra of the NIR 1, red, and green

409 bands are recovered according to Eq. (9), respectively. For each spectral band, the mean and

410 standard deviation (SD) in the non-shadow and shadow areas are summarized in Table 3 . The

411 shadow-restored imagery is depicted using a standard false color band combination (Fig. 6), as

412 well.

413

414 Table 3. Mean and SD of NIR 1, red, and green bands for the non-shadow and shadow areas.

\begin{tabular}{c|cccc}
\hline \multirow{2}{*}{ Spectral band } & \multicolumn{2}{|c}{ Non-shadow area } & \multicolumn{2}{c}{ Shadow area } \\
& Mean & Std. dev. & Mean & Std. dev. \\
\hline NIR 1 band & 311.80 & 151.49 & 73.10 & 43.22 \\
Red band & 227.02 & 126.08 & 70.17 & 31.08 \\
Green band & 408.63 & 181.30 & 179.79 & 46.00 \\
\hline
\end{tabular}

415
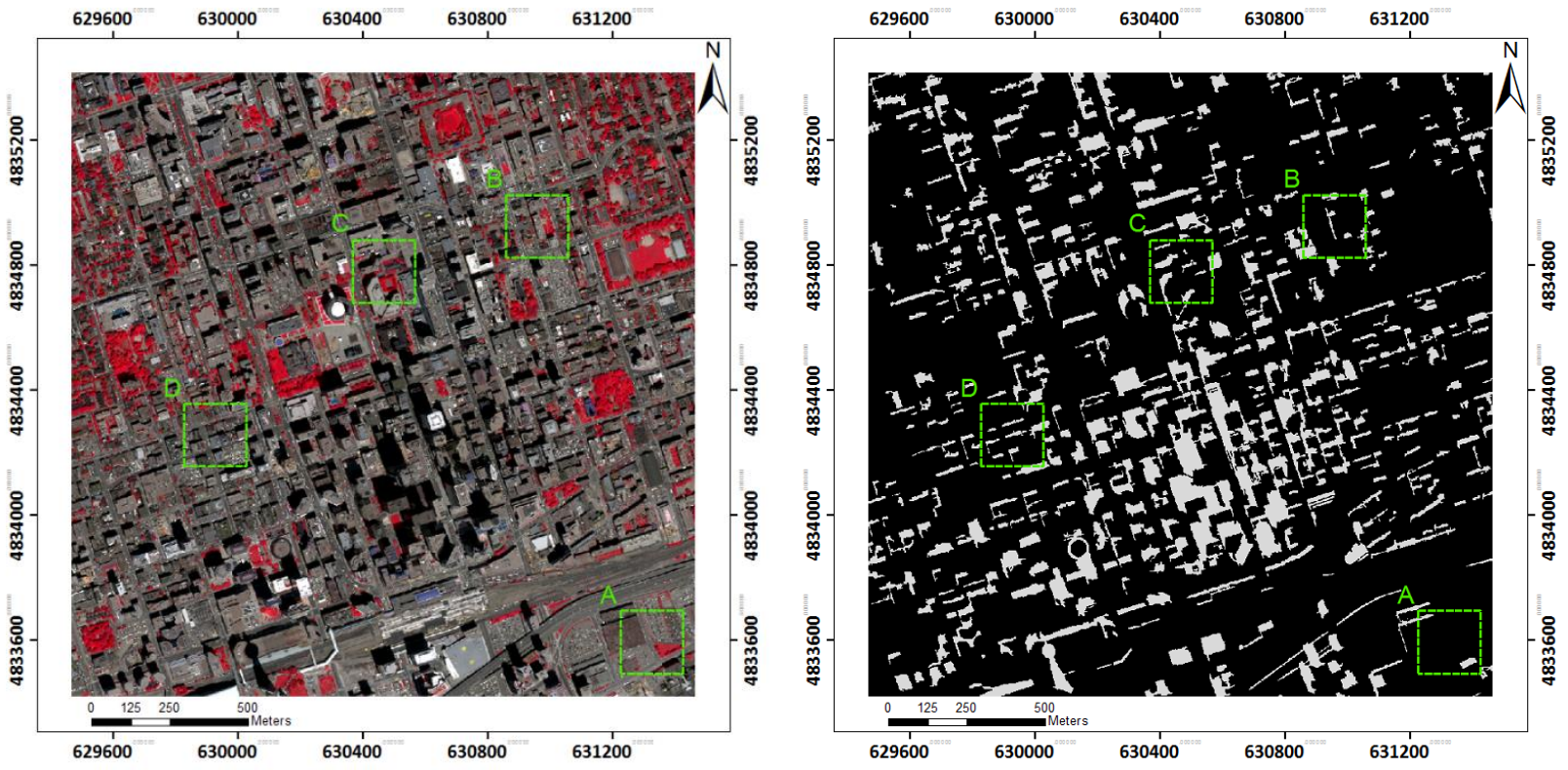

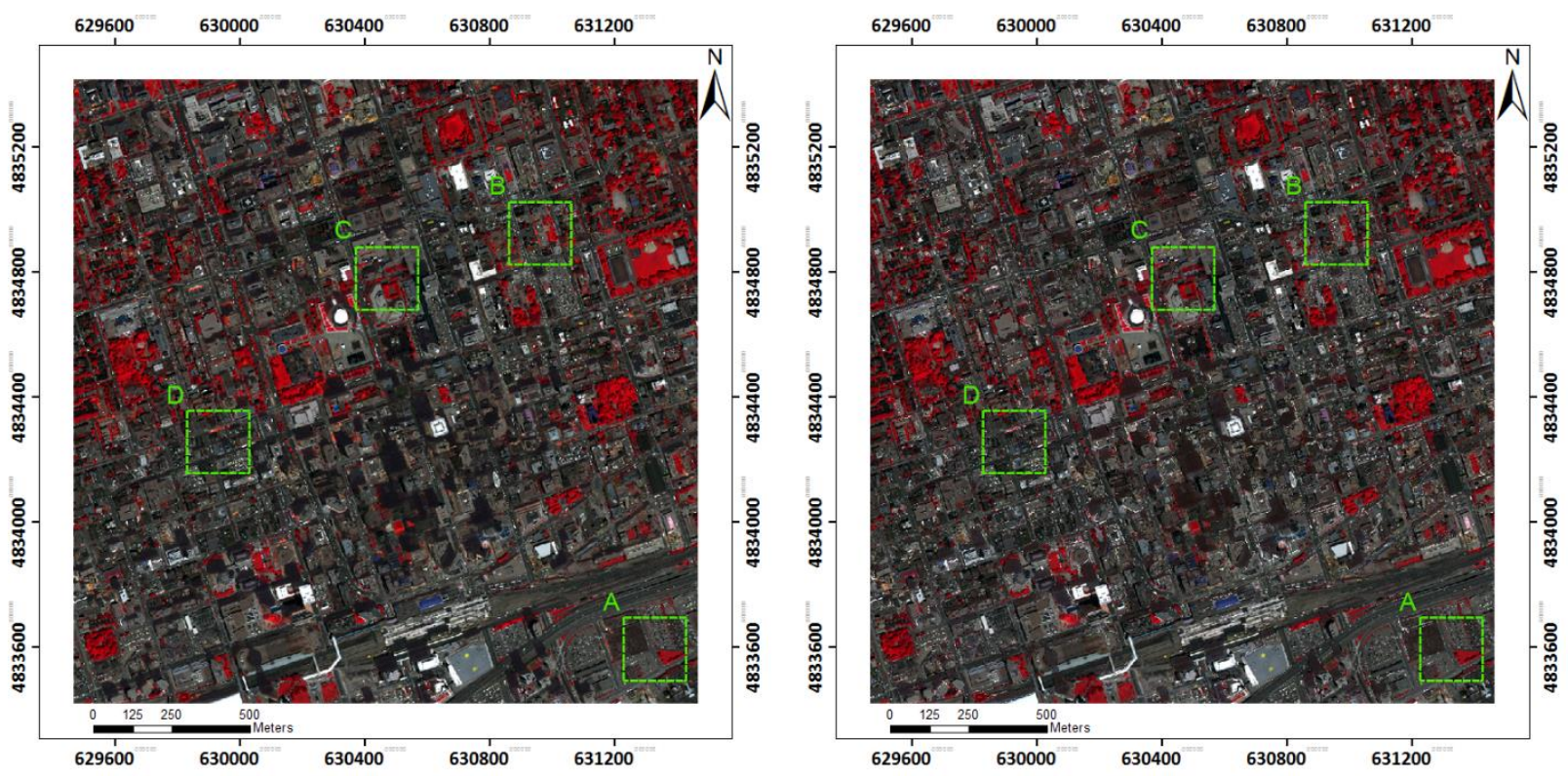

418 Fig. 6. Shadow compensation results of the entire experimental area (under WGS84 UTM

419 coordinate system). Upper left: original multispectral imagery; upper right: shadows (White);

420 lower left: fully constrained linear spectral unmixing based shadow-restored imagery; lower right:

421 linear-correlation correction based shadow-restored imagery. Green frames (A, B, C, and D) are

422 four subset areas from the entire imagery.

To compare differences in details, four small subsets of $100 \times 100$ pixels are randomly

425 chosen from the entire imagery (Green frame A, B, C, and D in Fig. 6) and zoomed in for visual

426 interpretation (Fig. 7). The shadow patches in the selected four subsets are labelled

427 alphabetically, most of which are pure shadow (i.e., single land cover) while a few of them are

428 compound shadow (i.e., multiple land covers), such as Fig. 7Ab, Bc, Bd, Bf, Cb, Cc, De, and Dh.

429 In contrast with the pure shadow patches, the compound shadow patches contain vegetation and

430 sealed surface (e.g., roads, rooftops, and parking lots), thus more important for urban land cover

431 classification. Overall, the proposed method produces the better shadow-restored imagery than

432 the conventional method in the above compound shadow patches. In the cases of Fig. 7Ab and 
$433 \mathrm{Cc}$, for instance, the vegetation information is better compensated and strengthened in the fully

434 constrained linear spectral unmixing based shadow-restored imagery. We also found that the

435 conventional linear-correlation correction method often overcompensates some pixels of sealed

436 surfaces. For example, the pixels on the edge of shadow patch shown as Fig. 7Ab are not so

437 evident and distinguishable from the others inside but these edge pixels are overcompensated to

438 the white. Moreover, the pixels surrounding vegetation in the cases shown as Fig. 7Bd and De

439 are very bright which is not reasonable. The proposed method overcomes this drawback and

440 compensates the shadow patches to a reasonable brightness. In addition to the compound shadow,

441 the shadow-restored imagery using the proposed method outperforms the other in terms of the

442 pure shadow because the restored spectral information is more similar to the adjacent non-

443 shadow land covers (Fig. 7Db, Dc, Dd, Df, Dg, and Di). Since the rationale of the proposed

444 method is to expand the scatter plot triangle of shadow area to the similar one of non-shadow

445 area, the shadow-restored imagery using the proposed method is able to retain the characteristics

446 of spectral mixing space better. Further, it is worth noting that the edges of shadow patches are

447 considerably distinct so that they are not continuous to the adjacent non-shadow land covers (Fig.

$4487 \mathrm{Cb}, \mathrm{Cc}$, and $\mathrm{Cg}$ ). This is the underlying flaw for the object-based shadow detection. In this

449 study, the proposed method refined the shadow-restored by smoothing the edge of non-shadow

450 and shadow areas which guarantees the continuity on the edge.

451
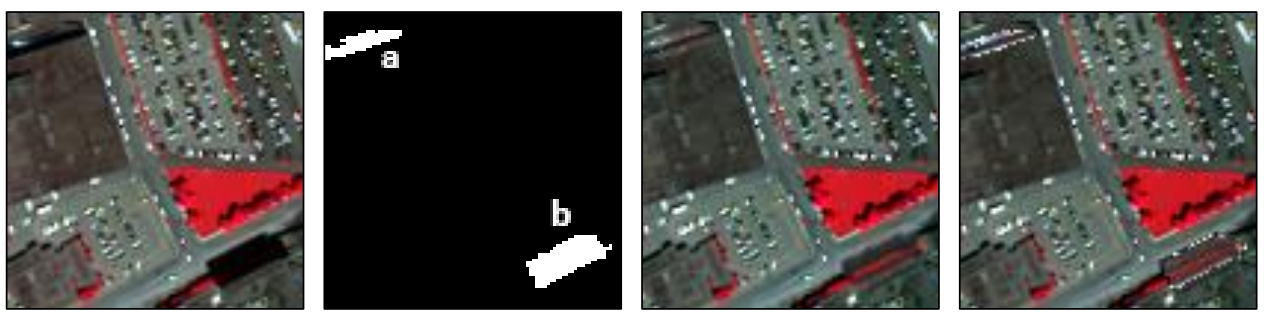

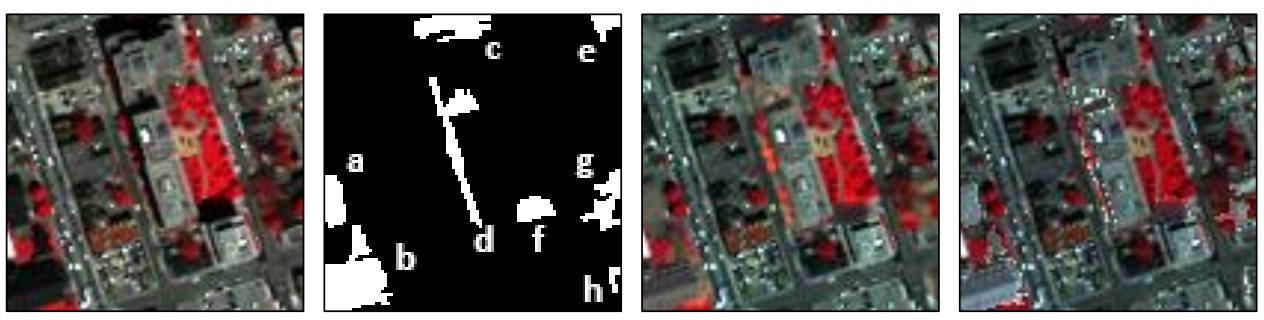

C
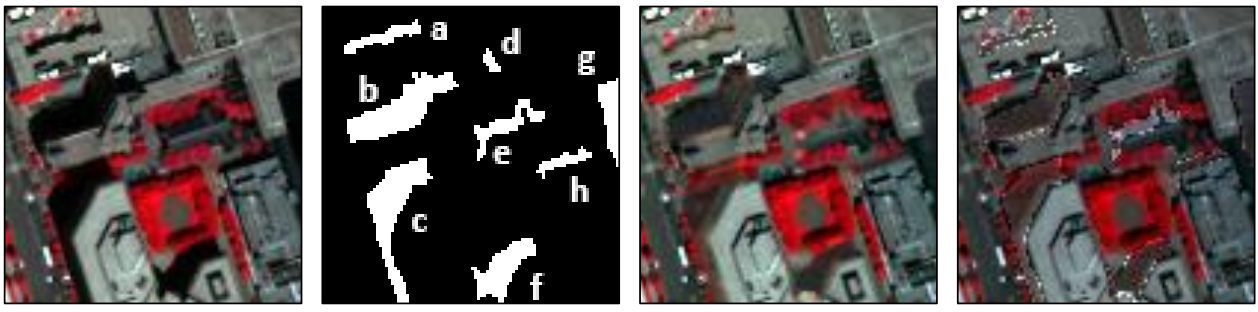

D
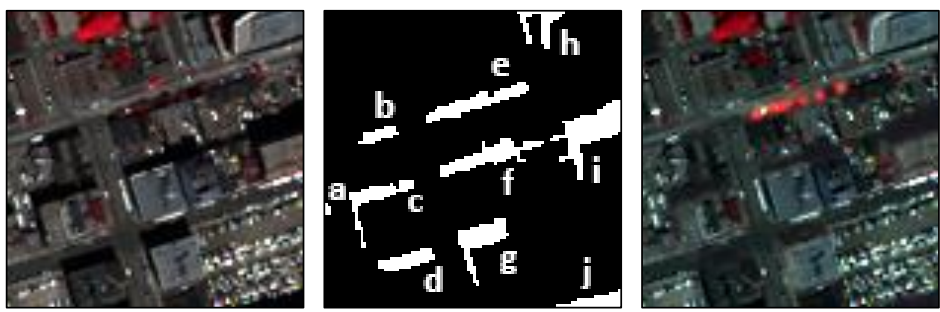

456

457 advantages of the proposed method over the conventional method. It is also implied that the success of the proposed global shadow compensation approach is mainly dependent upon two prerequisites. One is the accuracy of linear spectral unmixing, and the other one is the similarity

Fig. 7. An illustration of shadow compensation results in four subset areas A, B, C and D. From left to right: original imagery; shadow area (White); fully constrained linear spectral unmixing based shadow-restored imagery; linear-correlation correction based shadow-restored imagery.

\section{Discussion}

In terms of shadow compensation, the above visual inspection demonstrated the of the constructions of spectral scatter plots between the non-shadow and shadow areas.

The endmember fractions are used to characterize the spectral features of land covers in the shadow area, so inaccurate fractions can negatively impact the restoration of shaded surface 
materials. Moreover, endmember searching is one of the most crucial steps to accurately unmix endmember fractions. Most of the existing endmember searching methods were built upon representative selection within each independent class, such as EAR (Dennison and Roberts,

471 2003), MASA (Dennison et al., 2004), and CoB (Roberts et al., 2003). However, this popular

472 strategy is more appropriate to select training samples for supervised classifiers rather than to

473 select endmembers for linear spectral unmixing. More broadly, linear spectral unmixing is a kind

474 of image classification and endmember searching is also considered as generalized training 475 sample selection. Nonetheless, endmember searching has its typical and unique characteristics 476 because linear spectral unmixing is implemented within a spectral mixing space. Different from

477 the general selections of training samples, endmembers should be identified by investigating the 478 construction of spectral scatter plot, not focusing on only one class. Specifically, Yang et al.

479 (2014) elaborated that a triangle with straight edges is the optimal construction of a spectral 480 scatter plot for linear spectral mixing in a two-dimension spectral mixing space. Valid and 481 correct unmixed fractions could be derived when assigning three vertices of the triangle as 482 endmembers. Therefore, it is of great significance for linear spectral mixing to construct the 483 appropriate spectral mixing space so that the construction of the spectral scatter plot resembles 484 the optimal triangle, while the vertices represent the target endmembers. For example, we chose 485 three endmembers of vegetation, high albedo, and low albedo and further compared 12 two486 dimension spectral mixing spaces (Fig. 3) in which the vertices of the scatter plot triangles 487 represented these endmembers. In comparison with the other feature spaces, we implemented 488 endmember searching and linear spectral unmixing in the red-NIR 1 and green-NIR 1 spectral 489 mixing spaces because the more triangle-analogous spectral scatter plots indicated more accurate 490 unmixed fractions. In addition, we would like to clarify that linear spectral unmixing also differs 
491 from the common classifiers (e.g., maximum likelihood classifier, support vector machine,

492 random forest) in internal mechanisms. While these classifiers are implemented based on

493 statistical clustering, linear spectral unmixing is not. In other words, we cannot process linear

494 spectral unmixing as a solution for a mathematic problem. It has been already proved that linear

495 spectral unmixing is theoretically able to implement as long as the number of endmembers is not

496 greater than the number of spectral bands. Nevertheless, this prerequisite only ensures at least

497 one mathematic solution, but not the validity and accuracy. From this point of view, the analysis

498 of spectral scatter plot can provide the underlying foundation for endmember searching and

499 linear spectral mixing in a given spectral mixing space. Whenever unmixing multispectral or

500 hyperspectral imagery, it should be the highest priority to construct an appropriate spectral

501 mixing space (e.g., band math, transformation) for the endmembers of interest in order to

502 guarantee they locate at the vertices of a good scatter plot triangle.

503 The similarity of the constructions of spectral scatter plots between the non-shadow and

504 shadow areas is the basic hypothesis of the proposed shadow restoration method, which

505 representa an improvement on the assumption used by the conventional linear-correlation

506 correction method. In essence, the linear-correlation correction method adopts the similarity in

507 one-dimension spaces whereas the proposed method adopts the similarity in two-dimension

508 spaces. Obviously, two-dimension spaces characterize this similarity in more details than one-

509 dimension spaces, and thus can retain the construction of spectral scatter plot. On the other hand,

510 the linear-correlation correction is not a restoration process in the strict sense, although it is

511 widely known and utilized for shadow compensation. As the standard deviation of non-shadow

512 area $(\sigma(n))$ is always greater than that of shadow area $(\sigma(s))$, it is obvious to derive from Eq.

513 (9) that, 


$$
\frac{\stackrel{\Lambda}{D N}-\mu(n)}{D N-\mu(s)}=\frac{\sigma(n)}{\sigma(s)}>1(10)
$$

515 When the DN value of a shadow pixel $(D N)$ is greater than the mean value of the entire shadow 516 area $(\mu(s))$, Eq. (10) would be:

$$
\stackrel{\wedge}{D N}-\mu(n)>D N-\mu(s)(11)
$$

518 Since the mean DN value of non-shadow area $(\mu(n))$ is greater than that of shadow area $(\mu(s))$,

519 the restored $\mathrm{DN}$ value of this pixel $(\stackrel{\Lambda}{D N})$ must be greater than the original $\mathrm{DN}$ value $(D N)$.

520 However, when $D N$ is less than $\mu(s)$, Eq. (10) would become:

$$
\stackrel{\Lambda}{D N}-\mu(n)<D N-\mu(s)(12)
$$

522 We cannot conclude that $\stackrel{\Lambda}{D N}$ is greater than $D N$, thus we are not able to regard this process as 523 restoration in the strict sense. In some cases, the restored DN value is even less than the original 524 DN value. During the experiment, it was noticed that a few shaded pixels become negative when 525 implementing the conventional linear-correlation correction method. By contrast, the proposed 526 method restored the shadow pixels so that all the restored DN values were not less than the 527 original DN values in any spectral band. Despite that, the proposed method is not effective for 528 the restoration of pixels with a zero value (black shadows). Since 0-pixels fail to provide any 529 useful spectral information, we cannot calculate the endmember fractions and restore the spectral 530 information in those shadows. Although this problem can cause an incomplete shadow 531 restoration, the 0-pixels rarely exist in shadow areas over the actual imagery.

\section{Conclusions}


In this study, a novel global shadow compensation approach using fully constrained

535 linear spectral unmixing was proposed for high resolution satellite imagery of urban areas.

536 Compared to the conventional linear-correlation correction method, the proposed method

537 benefited the shadow-restored imagery in three aspects: 1) the compound shadow containing

538 vegetation and sealed surface was better restored and recovered without overcompensated pixels;

539 2) the characteristics of spectral mixing space were better retained so that the spectral properties

540 of restored shadow patches were quite similar to the adjacent land covers; and 3) the edge of

541 non-shadow and shadow areas was smoothed in order to ensure the continuity of land covers.

542 Although the proposed approach successfully improved shadow compensation for the

543 utilized WorldView-2 imagery, the existence of 0-pixels and the accuracy of shadow detection

544 could still limit its potential applications. In addition, the accuracy of object-based shadow

545 detection also strongly impacts the performance of the proposed compensation approach. The

546 misclassification of shadows and very dark rooftops can result in overcompensation of brightness

547 in the entire imagery. Therefore, further work will be conducted to eliminate the 0-pixels with

548 the assistance of local operators and to reduce the misclassification of shadows and very dark

549 rooftops in future work.

550

551 Acknowledgements

552 Support for this study was provided by NSERC Discovery Grant to Dr. Yuhong He in the 553 University of Toronto.

554

555 References 
Arbel, E., Hel-Or, H., 2011. Shadow removal using intensity surfaces and texture anchor points. IEEE Transactions on Pattern Analysis and Machine Intelligence 33, 1202-1216.

Benz, U.C., Hofmann, P., Willhauck, G., Lingenfelder, I., Heynen, M., 2004. Multi-resolution, object-oriented fuzzy analysis of remote sensing data for GIS-ready information. ISPRS Journal of Photogrammetry and Remote Sensing 58, 239-258.

Chen, Y., Wen, D., Jing, L., Shi, P., 2007. Shadow information recovery in urban areas from very high resolution satellite imagery. International Journal of Remote Sensing 28, 32493254.

Dare, P.M., 2005. Shadow analysis in high-resolution satellite imagery of urban areas. Photogrammetric Engineering \& Remote Sensing 71, 169-177.

Dennison, P.E., Halligan, K.Q., Roberts, D.A., 2004. A comparison of error metrics and constraints for multiple endmember spectral mixture analysis and spectral angle mapper. Remote Sensing of Environment 93, 359-367.

Dennison, P.E., Roberts, D.A., 2003. Endmember selection for multiple endmember spectral mixture analysis using endmember average RMSE. Remote Sensing of Environment 87, 123-135.

Graham, R.L., 1972. An efficient algorith for determining the convex hull of a finite planar set. Information processing letters 1, 132-133.

Li, H., Zhang, L., Shen, H., 2014. An Adaptive Nonlocal Regularized Shadow Removal Method for Aerial Remote Sensing Images. IEEE Transactions on Geoscience and Remote Sensing 52, 106-120.

Li, Y., Gong, P., Sasagawa, T., 2005. Integrated shadow removal based on photogrammetry and image analysis. International Journal of Remote Sensing 26, 3911-3929. 
579 Lorenzi, L., Melgani, F., Mercier, G., 2012. A complete processing chain for shadow detection and reconstruction in VHR images. IEEE Transactions on Geoscience and Remote Sensing 50, 3440-3452.

582

583

584

585

586

587

588

589

590

591

592

593

594

595

596

597

598

599

600
Massalabi, A., He, D.-C., Benie, G.B., Beaudry, E., 2004. Detecting information under and from shadow in panchromatic Ikonos images of the city of Sherbrooke, Proc. IEEE IGARSS. IEEE, pp. 2000-2003.

Nichol, J., Wong, M., 2007. Remote sensing of urban vegetation life form by spectral mixture analysis of high - resolution IKONOS satellite images. International Journal of Remote Sensing 28, 985-1000.

Nichol, J.E., Wong, M.S., Corlett, R., Nichol, D.W., 2010. Assessing avian habitat fragmentation in urban areas of Hong Kong (Kowloon) at high spatial resolution using spectral unmixing. Landscape and Urban Planning 95, 54-60.

Ridd, M.K., 1995. Exploring a VIS (vegetation-impervious surface-soil) model for urban ecosystem analysis through remote sensing: comparative anatomy for cities $\uparrow$. International Journal of Remote Sensing 16, 2165-2185.

Roberts, D.A., Dennison, P.E., Gardner, M.E., Hetzel, Y., Ustin, S.L., Lee, C.T., 2003. Evaluation of the potential of Hyperion for fire danger assessment by comparison to the Airborne Visible/Infrared Imaging Spectrometer. IEEE Transactions on Geoscience and Remote Sensing 41, 1297-1310.

Rogge, D.M., Rivard, B., Zhang, J., Feng, J., 2006. Iterative spectral unmixing for optimizing per-pixel endmember sets. IEEE Transactions on Geoscience and Remote Sensing 44, $3725-3736$. 
Sarabandi, P., Yamazaki, F., Matsuoka, M., Kiremidjian, A., 2004. Shadow detection and radiometric restoration in satellite high resolution images, Proc. IEEE IGARSS, pp. $3744-3747$.

Shahtahmassebi, A., Yang, N., Wang, K., Moore, N., Shen, Z., 2013. Review of shadow detection and de-shadowing methods in remote sensing. Chinese Geographical Science $23,403-420$.

Small, C., 2003. High spatial resolution spectral mixture analysis of urban reflectance. Remote Sensing of Environment 88, 170-186.

Somers, B., Asner, G.P., Tits, L., Coppin, P., 2011. Endmember variability in spectral mixture analysis: A review. Remote Sensing of Environment 115, 1603-1616.

Tsai, V.J., 2006. A comparative study on shadow compensation of color aerial images in invariant color models. IEEE Transactions on Geoscience and Remote Sensing 44, 16611671.

Van Der Meer, F., De Jong, S., 2000. Improving the results of spectral unmixing of Landsat Thematic Mapper imagery by enhancing the orthogonality of end-members. International Journal of Remote Sensing 21, 2781-2797.

Van der Meer, F.D., Jia, X., 2012. Collinearity and orthogonality of endmembers in linear spectral unmixing. International Journal of Applied Earth Observation and Geoinformation 18, 491-503.

Veraverbeke, S., Somers, B., Gitas, I., Katagis, T., Polychronaki, A., Goossens, R., 2012. Spectral mixture analysis to assess post-fire vegetation regeneration using Landsat Thematic Mapper imagery: Accounting for soil brightness variation. International Journal of Applied Earth Observation and Geoinformation 14, 1-11. 
624 Yamazaki, F., Liu, W., Takasaki, M., 2009. Characteristics of shadow and removal of its effects 625 for remote sensing imagery, Proc. IEEE IGARSS. IEEE, pp. IV-426-IV-429.

626 Yang, J., He, Y., Oguchi, T., 2014. An endmember optimization approach for linear spectral

627 unmixing of fine-scale urban imagery. International Journal of Applied Earth

628 Observation and Geoinformation 27, 137-146.

629 Zare, A., Ho, K., 2014. Endmember Variability in Hyperspectral Analysis: Addressing Spectral 630 Variability During Spectral Unmixing. Signal Processing Magazine, IEEE 31, 95-104.

631 Zhou, W., Huang, G., Troy, A., Cadenasso, M., 2009. Object-based land cover classification of 632 shaded areas in high spatial resolution imagery of urban areas: A comparison study.

633 Remote Sensing of Environment 113, 1769-1777.

634 